\title{
EXPERT ASSESSMENT OF THE SUITABILITY OF TREATMENT AND DIAGNOSTIC MEASURES IN PROVIDING EMERGENCY CARE TO PATIENTS WITH ACUTE CORONARY SYNDROME
}

\author{
Victor Zabashta, Bogdan Fedak
}

It is known that it is possible to affect the extent of myocardial damage and, as a result, mortality only in the first hours of its development. Therapeutic tactics in ACS with elevation of the ST segment involves the restoration of coronary blood flow, the main method is the reperfusion of the coronary artery by systemic thrombolysis or PCI in a specialized hospital. The effectiveness of treatment is inversely related to the time spent.

The aim of the study was to examine the opinion of specialists on the feasibility of implementing the items of the protocol of emergency medical care for patients with ACS at the place of call.

Materials and methods. During the study the method of expert evaluations, which consisted of gathering information by interviewing experts and summarizing the individual opinions of experts into a general concept was used. The experts were 48 emergency physicians. The method of expert evaluation included the following stages: development of the questionnaire; survey of experts; summary of examination materials; calculation of statistical indicators; interpretation of the obtained results and formulation of conclusions.

Results. In order to assess the actions of the emergency team, depending on the need to conduct them for diagnosis and emergency care for patients with ACS at the scene, we calculated the feasibility indexes for each item of the protocol. In the future, we divided the treatment and diagnostic measures for ACS with ST segment elevation according to the level of expediency at the scene into four groups (n): $n_{1}$ - high level, $n_{2}-$ sufficient level and $n_{3}-$ low level and $n_{4}-v e r y$ low level. According to the results of the ranking, the scope of measures to be implemented by the head of the emergency team at the ACS with elevation of the ST segment on arrival on call, as well as measures that, according to interviewed experts, are not required at this stage and can be carried out during transportation of the patient to a specialized hospital.

Conclusions. Based on the results of the calculation of feasibility indices and subsequent ranking of treatment and diagnostic measures for the relevant groups $\left(n_{1}, n_{2}, n_{3}, n_{4}\right)$ from 37 items of the study, to assist patients with ACS with ST segment elevation at the scene, it is recommended to perform 16

Keywords: acute coronary syndrome, method of expert assessments, medical and diagnostic measures, prehospital stage

How to cite:

Zabashta, V., Fedak, B. (2021). Expert assessment of the suitability of treatment and diagnostic measures in providing emergency care to patients with acute coronary syndrome. ScienceRise: Medical Science, 3 (42), 38-44. doi: http://doi.org/10.15587/2519-4798.2021.232978

(C) The Author(s) 2021

This is an open access article under the Creative Commons CC BY license

\section{Introduction}

The influence of various factors on the course and prognosis in patients with acute coronary syndrome (ACS) with ST segment elevation has been proven [1,2]. Such factors include demographic indicators, such as gender, age [3], anamnesis data (death of parents from heart disease, self-administration of antianginal drugs $[3,4]$, hemodynamic parameters), blood pressure, heart rate $[5,6]$, etiology and pathogenesis (nature of coronary artery occlusion, severity of coronary artery disease) [7], time factors (therapeutic window up to $12 \mathrm{~h}$ from the moment of clinical symptoms of the disease or a time interval up to $24 \mathrm{~h}$ from the moment of development of the clinical picture of ACS for percutaneous coronary interventions (PCI) $[8,9]$.

A patient with ACS who was hospitalized in a specialized cardiac hospital for the first 90 minutes after the onset of an angina attack has a better chance of success [2]. The results of the analysis of time indicators of patient care at the prehospital stage suggest that 90 minutes should include the time from the onset of pain to the receipt and processing of the call by the emergency department of the emergency medical service (EMS), the time of transfer of the call to the EMS, time spent on providing emergency medical care at the scene, time of transportation of the patient to a specialized health care institution $[6,10,11]$.

It should be noted that the waiting time of the EMS team for more than 20 minutes for a patient with ACS is characterized by the beginning of the breakdown of mitochondria of damaged cardiomyocytes, which with a delay of 70 minutes increases by $50 \%$, and at 150 minutes - vascular insufficiency doubles $[6,10,12]$. As a result, delays in the health system increase the relative risk of death and the development of acute cardiovascular failure.

That is why the European guidelines state that no more than 10 minutes should pass from the time of the 
first contact of the health worker with the patient to the ECG, no more than 30 minutes from the time of the first contact to the reperfusion therapy and no more than 90 minutes to the delivery of the patient to the catheterization laboratory [13].

It should be noted that the current protocols of EMS crews at ACS in Ukraine provide a number of medical and diagnostic measures, including collection of medical history and life history, examination and physical examination, assessment of cardiovascular and respiratory systems, instrumental examination, actual treatment $[14,15]$. The full implementation of this algorithm of measures requires a long time, which can lead to the impossibility of starting early reperfusion therapy in a specialized hospital.

Since the introduction of medical reform in late 2018 in the Kharkiv region, many issues of organization of the emergency medical service, significantly reduced and brought to world standards, the time of arrival of teams on call, measures taken to organize traffic.

However, the issue of optimizing the measures needed to diagnose and provide emergency care to patients with ACS in the prehospital phase in order to reduce delivery time to receive specialized/highly specialized care in the hospital remains unresolved.

The aim of the study was to examine the opinion of experts on the feasibility of implementing the items of the protocol of emergency medical care for patients with ACS at the scene.

\section{Materials and methods}

The study used the method of expert assessments, which was conducted in the period from 25/11/2018 to 24/12/2018 and consisted of gathering information by interviewing experts and summarizing the individual opinions of experts into a general concept. The experts were 48 health care organizers and scientists from different regions of Ukraine. The expert group was formed on professional grounds and consisted of highly qualified emergency physicians with practical experience in the organization of health care, directly involved in the medical-diagnostic process and functional responsibilities related to the organization of the medical-diagnostic process, requirements for which were the presence of a degree and work experience of at least 20 years. Thus, the group of experts consisted of 16 people with the title of doctor of medicine, 22 - doctor of philosophy, $10-$ health care organizers, which certifies the degree of professionalism, competence, as well as the reliability and objectivity of the assessment.

The reliability of the obtained information from expert assessments is ensured by the appropriate amount of the research. To determine the number of experts to be covered by the study, the recommendations of O. S. Ventzel were used, according to which to determine the minimum number of experts in medical research, it is advisable to use the marginal error $\Delta=0.5-0.3$ with a confidence interval of 90-95\%. Thus, with a marginal error $\Delta=0.3$ and a confidence level of $90 \%$, the tabular value of the minimum number of experts, calculated by $\mathrm{O}$. S. Ventzel, is 31 . Thus, our sample of 48 experts can be considered representative for expert evaluation $[15,16]$.

\section{Research results}

To conduct an expert survey of physicians, a questionnaire was developed in the form of a table with a list of actions of the EMS team, which were evaluated by experts on the criterion: "appropriate when calling" (yes / no / possible). The list of medical and diagnostic measures that were included in the questionnaire for expert evaluation was based on the protocol of medical care for patients with ACS of the Ministry of Health of Ukraine [14].

In order to evaluate the actions of the EMS team, depending on the need for them to diagnose and provide emergency care to patients with ACS, an evaluation scale from 0 to 2 points was used:

-0 points - impractical;

-1 point - possible;

-2 points - appropriate.

As a result of processing the questionnaires, we calculated the indices of expediency for each item of the protocol according to formula 1 [17]:

$$
I=\frac{2 * a+b+0 * c}{N},
$$

where $I$-index of expediency for each studied parameter; $a, b, c$ - the number of experts who chose a certain parameter; $N$ - total number of respondents.

The results of the calculations are given in Table 1.

According to the results of the calculations, among the studied therapeutic and diagnostic measures, which according to the protocol are recommended for ACS with ST-segment elevation, the most appropriate at the scene of the call, according to experts, were actions whose feasibility index was 96 , including "Set accurate time from the onset of chest pain and its duration", "Assessment of general condition and vital functions: consciousness, respiration, blood circulation according to the ABCDE algorithm", "Pulse, its characteristics", "ECG recording in 12 leads or transmission of biometric ECG signals to the consultative telemetry center for the decision of urgent questions of interpretation of an ECG", "Position of the patient lying down with the raised slightly head", "Limit physical activity, ensure complete psychological peace", "Providing venous access (catheterization of the peripheral vein)" and "Nitroglycerin under the tongue in tablets or aerosol.

In case of severe pain - intravenous drip under constant control of blood pressure and heart rate". 
Table 1

The results of the respondents' assessment of the feasibility of treatment and diagnostic measures for ACS with elevation of the ST segment at the call site

Actions

\begin{tabular}{|c|c|c|c|}
\hline \multicolumn{2}{|c|}{$\begin{array}{c}\text { Proportion of experts who } \\
\text { chose the parameter, \% }\end{array}$} & $\begin{array}{c}\text { Expedi- } \\
\text { ency } \\
\text { index }\end{array}$ \\
\hline yes & no & possible & ind \\
\hline
\end{tabular}

1.1. Collection of medical history:

1.1.1. Establish the exact time from the beginning of the attack of chest pain and its duration.

1.1.2. Establish the nature of pain, its localization and irradiation.

1.1.3. Determine if there was an attempt to relieve the pain with nitroglycerin.

1.1.4. Determine the conditions under which pain occurs - whether it is associated with physical, psycho-emotional stress.

1.1.5. Find out if there were attacks of pain or suffocation while walking, or if you were forced to stop, their duration in minutes. Were these attacks relieved by nitroglycerin.

1.1.6. Is this attack of pain or suffocation similar to the sensations that occurred earlier during exercise in terms of location and nature.

1.1.7. Has the pain gotten worse and more frequent lately. It is desirable to indicate whether the load tolerance has changed or the need for nitrates has increased.

1.2. Collection of life history:

1.2.1. Determine which drugs the patient takes daily.

1.2.2. Find out which drugs the patient took before the arrival of the EMS team.

1.2.3. Find out the presence of CVD risk factors. Identify a history of other comorbidities.

1.2.4. Collect a general history of allergies and find out if there are any allergic reactions to the drug.

2. General examination and physical examination

2.1. Assessment of general condition and vital functions: consciousness, respiration, blood circulation according to the ABCDE algorithm.

2.2. According to the indications to eliminate violations of vital functions of the body respiration, blood circulation.

2.3. Visual assessment:

2.4. The color of the skin, moisture, swelling of the jugular veins.

2.5. Blood pressure measurement on both arms.

2.6. Percussion of the heart: pay attention to the presence of an increase in the limits of cardiac dullness.

2.7. Palpation of the heart: to assess the apex beat and its location.

2.8. Auscultation of the heart and blood vessels: assess the tone and presence of noise, the presence of III heart tone or IV heart tone; rhythmic heartbeat.

2.9. Pulse, its characteristics.

2.10. HR, its characteristics.

2.11. Pulmonary auscultation: the presence of wheezing.

2.12. Palpation of the abdominal organs: enlargement of the liver.

3. Conducting an instrumental survey

3.1. ECG recording in 12 leads or transmission of biometric ECG signals to a telemetrycounselling center to address urgent issues of ECG interpretation.

3.2. If at the beginning of clinical manifestations of AMI there are no electrocardio-

graphic signs, ECG registration should be repeated with an interval of 20-30 minutes.

3.3. Pulse oximetry (determination of blood oxygenation by oxygen).

3.4. Determination of elevated levels of cardiomarkers (Troronin I and Troronin T, SK-

MV, Myoglobin) in the blood using a kit for rapid diagnosis.

Therapeutic tactics

1. The position of the patient lying down with his head slightly raised. Limit physical activity, provide complete psychological peace.

2. Oxygen therapy. Inhalation of humidified oxygen should be performed using a mask or through a nasal catheter.

3. Providing venous access (peripheral vein catheterization).

4. Nitroglycerin under the tongue in tablets or aerosol. In case of severe pain - intravenous drip under constant control of blood pressure and heart rate.

5. Acetylsalicylic acid (used if the patient did not take it on his own before the arrival of the EMS team).

6. Clopidogrel $300 \mathrm{mg}$ orally.

7. Narcotic analgesics.

8. Beta-blockers are prescribed to all patients with AMI who have no contraindications.

9. Statins are recommended to be prescribed or continued in high doses.

10. Enoxaparin intravenously and after 15 minutes additionally subcutaneously or heparin - intravenously.

11. Blood pressure control and correction.

\begin{tabular}{|c|c|c|c|c|}
\hline 100 & - & - & 96 \\
\hline 83.3 & 10.4 & 6.3 & 83 \\
\hline 22.9 & 31.3 & 45.8 & 44 \\
\hline 45.8 & 27.1 & 27.1 & 57 \\
\hline 20.8 & 50.0 & 29.2 & 34 \\
\hline 18.8 & 60.4 & 20.8 & 28 \\
\hline 18.8 & 60.4 & 20.8 & 28 \\
\hline
\end{tabular}

\begin{tabular}{|c|c|c|c|c|}
\hline & 18.8 & 47.9 & 33.3 & 34 \\
\hline 68.7 & 0.0 & 31.3 & 81 \\
\hline & 39.6 & 33.3 & 27.1 & 51 \\
\hline & 68.8 & 20.8 & 10.4 & 71 \\
\hline
\end{tabular}

\begin{tabular}{|c|c|c|c|c|}
\hline 100 & - & - & 96 \\
\hline 93.8 & - & 6.2 & 93 \\
\hline 77.1 & 8.3 & 14.6 & 81 \\
\hline 52.1 & 35.4 & 12.5 & 56 \\
\hline 14.6 & 77.1 & 8.3 & 18 \\
\hline 16.7 & 62.5 & 20.8 & 26 \\
\hline 66.7 & 8.3 & 25.0 & 76 \\
\hline 100 & - & - & 96 \\
\hline 79.2 & 4.2 & 16.7 & 84 \\
\hline 75.0 & 2.1 & 22.9 & 83 \\
\hline 20.8 & 41.7 & 37.5 & 38 \\
\hline
\end{tabular}

20.8

\begin{tabular}{|c|c|c|c|c|}
\hline 100 & - & - & 96 \\
\hline 16.6 & 79.2 & 4.2 & 18 \\
\hline 29.2 & 45.8 & 25.0 & 40 \\
\hline 20.8 & 66.7 & 12.5 & 26 \\
\hline
\end{tabular}

\begin{tabular}{|c|c|c|c|c|}
\hline 97.9 & - & 2.1 & 95 \\
\hline 37.5 & 39.6 & 22.9 & 47 \\
\hline 100 & - & - & 96 \\
\hline 100 & - & - & 96 \\
\hline 31.3 & 62.5 & 6.3 & 33 \\
\hline 14.6 & 81.3 & 4.2 & 16 \\
\hline 68.8 & 4.2 & 27.1 & 79 \\
\hline 37.5 & 20.8 & 41.7 & 56 \\
\hline 20.8 & 8.3 & 70.8 & 54 \\
\hline 27.1 & 31.2 & 41.7 & 46 \\
\hline 16.7 & 79.2 & 4.2 & 18 \\
\hline
\end{tabular}


Experts consider "Percussion of the heart: pay attention to the presence of an increase in the limits of cardiac dullness", "If there are no electrocardiographic signs at the beginning of clinical manifestations of AMI", "ECG recording should be repeated at intervals of 2030 minutes" and "Control and correction of blood pressure" (index of expediency of which was 18), "Clopidogrel $300 \mathrm{mg}$ orally" (index of expediency - 16).

Subsequently, to rank the treatment and diagnostic measures into groups on the feasibility of their implementation in the provision of emergency care to patients with ACS in the prehospital stage, all studied parameters were divided into 2 sectors for which the value of the feasibility index is greater or less than the arithmetic mean, calculated by formula 2 [17]:

$$
I_{a v}=\frac{1}{n} \cdot \sum_{i=1}^{n} I_{i},
$$

where $I_{\mathrm{av}}$ - the arithmetic mean of the expediency index; $\mathrm{n}$ - the number of studied parameters.

The calculated arithmetic mean value of the indices of expediency of medical and diagnostic measures at the place of call is 59. The first sector includes items of the protocol, the index of expediency of which was greater than the arithmetic mean of the indices of expediency of the studied parameters; the second sector includes items of the protocol, the index of expediency of which was less than the arithmetic mean.

Based on the results of $I_{\text {av }}$ calculations, we ranked treatment and diagnostic measures for ACS with elevation of the ST segment according to the level of expediency of conducting on-site events into four groups (n): $n_{1}-$ high level, $n_{2}-$ sufficient level and $n_{3}-$ low level and $n_{4}-$ very low level. In order to distribute the studied parameters, we used the law of variation of individual values of the feature, in particular, the "rule of three sigma". According to this rule, we calculated the standard deviation of the index of expediency of the studied parameters in each of the sectors according to formulas 3 and 4 [17]:

$$
\begin{aligned}
& \sigma_{1}=\sqrt{\left(\frac{1}{k}\right) \cdot \sum_{s=1, \ldots, k}^{k}\left(I_{i(s)}-I_{a v 1}\right)^{2}}, \\
& \sigma_{2}=\sqrt{\frac{1}{n-k} \cdot \sum_{t=1, \ldots, n-k}^{n-k}\left(I_{i(t)}-I_{a v 2}\right)^{2}},
\end{aligned}
$$

where $I_{i(S)}$ - index of expediency of the studied parameters for which $I_{i(S)} \geq I_{a v}$;

$I_{i(t)}$ - index of expediency of the studied parameters for which $I_{i(t)}<I_{a v}$;

$k$ - the number of parameters for which $I_{i(S)} \geq I_{a v}$;

$(n-k)-$ the number of parameters for which $I_{i(t)}<I_{a v}$;

$I_{a v .1}-$ the arithmetic mean of the expediency indices for which $I_{i(S)} \geq I_{a v}$;

$I_{a v .2}-$ the arithmetic mean of the expediency indices for which $I_{i(t)}<I_{a v}$.

The calculated standard deviations of the expediency indices of the studied parameters when arriving on call by the first and second sectors, which were $\sigma_{1=8,38}$ and $\sigma_{2}=13,46$, respectively, taking into account the maximum and minimum values allow to determine the boundaries of classification groups (Table 2).

Table 2

The results of the distribution of therapeutic and diagnostic measures in ACS with elevation of the ST segment on the feasibility of performing at the call site

\begin{tabular}{|l|c|c|c|}
\hline $\begin{array}{c}\text { Classification groups of medical and diagnostic } \\
\text { measures according to the level of expediency }\end{array}$ & $\begin{array}{c}\text { Criteria of expedi- } \\
\text { ency }\end{array}$ & $\begin{array}{c}\text { Ranges of change of } \\
\text { expediency indices }\end{array}$ & $\begin{array}{c}\text { Number of pro- } \\
\text { tocol items }\end{array}$ \\
\hline High & {$\left[I_{\max } ; \ldots I_{a v}+3 \times \sigma_{1}\right]$} & {$[96 ; \ldots 84]$} & 9 \\
\hline Sufficient & {$\left[I_{a v}+3 \times \sigma_{1} ; \ldots I_{a v}\right]$} & {$[84 ; \ldots 59]$} & 7 \\
\hline Low & {$\left[I_{a v} ; \ldots I_{a v}-3 \times \sigma_{2}\right]$} & {$[59 ; \ldots 18]$} & 17 \\
\hline Very low & {$\left[I_{a v}-3 \times \sigma_{2} ; \ldots I_{\min }\right]$} & {$[18 ; \ldots 16]$} & 4 \\
\hline
\end{tabular}

Note: $I_{\max }-$ the maximum value of the feasibility index; $I_{\min }-$ the minimum value of the feasibility index

According to the results of the division of actions of the EMS team leader into groups according to the calculated feasibility index, it was established that the first classification group (medical and diagnostic measures with a high level of feasibility) with a range of feasibility index from 96 to 84 included 9 protocol items. The second group (medical and diagnostic measures with a sufficient level of feasibility) includes 7 items of the protocol, for which the fluctuations of the feasibility index ranged from 84 to 59. The third group of actions of the head of the EMS team (medical and diagnostic measures with a low level of expediency) contains 17 items of the protocol with the value of the feasibility index from 59 to 18. The fourth group of actions (medical and diagnostic measures with a very low level of feasibility) with char- acteristic fluctuations of the feasibility index in the range from 18 to 16 , formed from 4 items of the protocol.

Thus, the list of treatment and diagnostic measures that are necessary to provide emergency care to patients with ACS with elevation of the ST segment at the scene, included 16 points of the protocol (out of the 37 parameters studied) groups $n_{1}$ and $n_{2}$, which had values of the expediency index from 96 to 59 .

According to the results of the study, we have identified the scope of measures to be implemented by the head of the EMS team at ACS patient with elevation of the ST segment on call, as well as measures that, according to interviewed experts, are not mandatory at this stage and can be implemented during transportation of the patient to a specialized hospital (Fig. 1). 


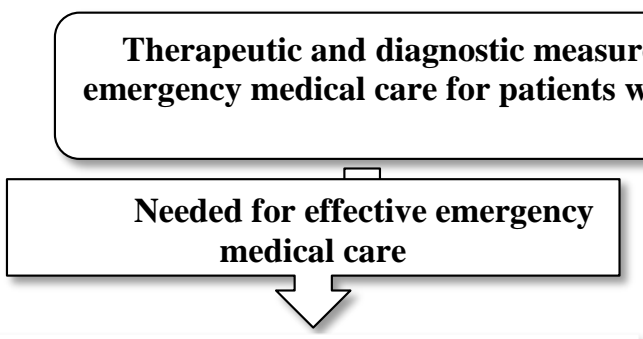

- Set the exact time from the onset of chest pain and its duration.

- Establish the nature of pain, its localization and irradiation.

- Determine the conditions under which the pain occurs - whether it is associated with physical and emotional stress.

- Collect a general history of allergies and find out if there are any allergic reactions to the drug.

- Find out which drugs the patient has taken before the arrival of the EMS team.

- Assessment of general condition and vital functions: consciousness, respiration, blood circulation according to the $\mathrm{ABCDE}$ algorithm.

- In accordance with the indications to eliminate violations of vital functions of the body - breathing, blood circulation.

- Assessment of skin color, moisture, swelling of the jugular veins.

- Pulse, its characteristics.

- $\mathrm{BH}$, its characteristics.

- Auscultation of the lungs: the presence of wheezing.

- 12-lead ECG recording or transmission of biometric ECG signals to a telemetry counseling center to address urgent ECG interpretation issues.

- Pulse oximetry (determination of oxygen oxygenation of the blood).

- The position of the patient lying down with his head slightly raised. Limit physical activity, provide complete psychological peace.

- Providing venous access (catheterization of the peripheral vein).

- Nitroglycerin under the tongue in tablets or in aerosol. In case of severe pain - intravenous drip under constant control of blood pressure and heart rate.

- Narcotic analgesics.
Are impractical at this stage

- Determine if an attempt has been made to relieve the pain with nitroglycerin.

- Find out if there were bouts of pain or suffocation while walking, or if they were forced to stop, their duration in minutes. Were these attacks relieved by nitroglycerin.

- Is this attack of pain or suffocation similar to the sensations that occurred earlier during exercise in terms of location and nature.

- Has the pain gotten worse and more frequent lately? It is desirable to indicate whether the load tolerance has changed or the need for nitrates has increased.

- Determine which drugs the patient is taking daily.

- Find out the presence of CVD risk factors.

Identify a history of other comorbidities.

- Measurement of blood pressure on both hands.

- Percussion of the heart.

- Palpation of the heart.

- Auscultation of the heart and blood vessels.

- Palpation of the abdominal organs: enlargement of the liver.

- If there are no electrocardiographic signs at the beginning of clinical manifestations of AMI,

ECG recording should be repeated at intervals of 20-30 minutes.

- Determination of elevated levels of

cardiomarkers (Troronin I and Troronin T, SK-

$\mathrm{MB}$, Myoglobin) in the blood using a kit for rapid diagnosis.

- Oxygen therapy.

- Acetylsalicylic acid.

- Clopidogrel 300 mg orally.

- Beta-blockers.

- Statins.

- Enoxaparin intravenously and after 15 minutes additionally subcutaneously or heparin -

intravenously.

- Control and correction of blood pressure.

Fig. 1. Generalization of the results of the survey of respondents on the feasibility of treatment and diagnostic measures for ACS with elevation of the ST segment at the call site.

\section{Discussion of research results}

Domestic recommendations for the diagnosis and treatment of patients with ACS with elevation of the ST segment are dated since 2014. The basics of diagnosis and treatment of these patients, set out in the clinical protocol in 2014, remain valid today [14]. However, in re- cent years, new data and new treatment and diagnostic approaches have emerged that allow more successful treatment of patients with ACS [1, 13]. Today, the importance of early contact of the patient with a health worker and the beginning of reperfusion therapy is not in doubt $[2,8]$. That is why the effective organization of 
treatment, close interaction of prehospital and subsequent stages of medical care becomes especially important.

The modern view of early therapeutic intervention in ACS in scientific publications in recent years is characterized by the concept of "golden hour", because it is timely provision of necessary medical care to the patient using thrombolytic therapy within the therapeutic window can prevent complications $[9,10$, 11]. Much can be done to reduce the time for prehospital treatment in order to provide the necessary medical care to patients in sufficient numbers and in the shortest possible time. In particular, at the prehospital stage it is necessary [4, 13].

- conduct public awareness campaigns - dissemination of information that has a simple and widely available form in order to widely inform the public to understand the symptoms and risk factors for heart attack and awareness of the need for measures to modify them;

- rapid identification of patients and response to signs characteristic of the onset of ACS;

- immediate dispatch of the EMS team;

- providing the necessary medical care using all available opportunities to stabilize the patient's condition; - presence of link between the EMS team and the hospital, which allows to warn about the arrival of a patient with acute cardiovascular disease.

An effective system for organizing EMS teams can minimize pre-hospital delays by reducing the time required to send, assess the patient's condition, provide emergency care and transport patients, which increases the number of people with a heart attack who are hospitalized and who are it is possible to conduct PCI in a time window of up to 3 hours.

Study limitations. The results of the analysis reflect the level of appropriateness of treatment and diag- nostic measures for ACS, available in the National Treatment Protocol, only health care providers and scientists who joined the study.

Prospects for further research. The results of the study can be used in the future to form a local protocol for emergency medical care at patients with ACS in the Kharkiv region.

\section{Conclusions}

Expert assessment is one of the main stages in the process of creating scientifically sound medical standards and unified clinical protocols. During the study, a questionnaire and an algorithm for expert assessment of the feasibility of treatment and diagnostic measures for ACS with elevation of the ST segment at the call site were developed.

The calculations of expediency indices for each item of the unified clinical protocol of emergency medical care in ACS with ST segment elevation allowed to rank the actions of the EMS team leader when arriving on call in four groups according to the level of expediency according to expert estimates.

Based on the results of the calculation of feasibility indices and subsequent ranking of treatment and diagnostic measures for the relevant groups $\left(\mathrm{n}_{1}, \mathrm{n}_{2}, \mathrm{n}_{3}, \mathrm{n}_{4}\right)$ of the 37 items of the study, to assist patients with ACS with ST segment elevation at the scene, it is recommended to perform 16 .

\section{Conflict of interests}

The authors declare that they have no conflicts of interest.

\section{Financing.}

None

\section{References}

1. Kawecki, D., Morawiec, B., Gąsior, M., Wilczek, K., Nowalany-Kozielska, E., Gierlotka, M. (2019). Annual Trends in Total Ischemic Time and One-Year Fatalities: The Paradox of STEMI Network Performance Assessment. Journal of Clinical Medicine, 8 (1), 78. doi: http://doi.org/10.3390/jcm8010078

2. Khalid, U., Jneid, H., Denktas, A. E. (2017). The relationship between total ischemic time and mortality in patients with STEMI: every second counts. Cardiovascular Diagnosis and Therapy, 7 (S2), S119-S124. doi: http://doi.org/10.21037/cdt.2017.05.10

3. Sederholm Lawesson, S., Isaksson, R.-M., Thylén, I., Ericsson, M., Ängerud, K., Swahn, E. (2018). Gender differences in symptom presentation of ST-elevation myocardial infarction - An observational multicenter survey study. International Journal of Cardiology, 264, 7-11. doi: http://doi.org/10.1016/j.ijcard.2018.03.084

4. Vertkin, A. L., Morozov, S. N., Fedorov, A. I. (2013). Prehospital stage of medical aid to patients with acute coronary syndrome and elevated ST segment. Klinicheskaya meditsina, 7, 65-69.

5. Kontsevaya, A. V., Kalinina, A. M., Koltunov, I. E., Oganov, R. G. (2011). Sotsialnoekonomicheskiy uscherb ot ostrogo koronarnogo sindroma v Rossiyskoy Federatsii. Ratsionalnaya farmakoterapiya v kardiologii, 7, 158-166.

6. Dracup, K., McKinley, S., Riegel, B., Moser, D. K., Meischke, H., Doering, L. V. et. al. (2009). A Randomized Clinical Trial to Reduce Patient Prehospital Delay to Treatment in Acute Coronary Syndrome. Circulation: Cardiovascular Quality and Outcomes, 2 (6), 524-532. doi: http://doi.org/10.1161/circoutcomes.109.852608

7. Veselova, T. N., Merkulova, I. N., Barysheva, N. A., Ternovoy, S. K., Shariya, M. A., Ruda, M. Ya. (2013). Comparison of characteristics of atherosclerotic plaques in patients with acute coronary syndrome and stable ischemic heart disease: data of multispiral computed tomography. Kardiologiya, 53 (12), 14-20.

8. Nielsen, C. G., Laut, K. G., Jensen, L. O., Ravkilde, J., Terkelsen, C. J., Kristensen, S. D. (2016). Patient delay in patients with ST-elevation myocardial infarction: Time patterns and predictors for a prolonged delay. European Heart Journal: Acute Cardiovascular Care, 6 (7), 583-591. doi: http://doi.org/10.1177/2048872616676570

9. Denktas, A. E., Anderson, H. V., McCarthy, J., Smalling, R. W. (2011). Total Ischemic Time: the correct focus of attention for optimal ST-segment elevation myocardial infarction care. JACC: Cardiovascular Interventions, 4 (6), 599-604. doi: http://doi.org/10.1016/j.jcin.2011.02.012 
10. Foo, C. Y., Bonsu, K. O., Nallamothu, B. K., Reid, C. M., Dhippayom, T., Reidpath, D. D., Chaiyakunapruk, N. (2018). Coronary intervention door-to-balloon time and outcomes in ST-elevation myocardial infarction: a meta-analysis. Heart, 104 (16), 1362-1369. doi: http://doi.org/10.1136/heartjnl-2017-312517

11. Kim, H. K., Jeong, M. H., Ahn, Y., Chae, S. C., Kim, Y. J., Hur, S. H. et. al. (2017). Relationship between time to treatment and mortality among patients undergoing primary percutaneous coronary intervention according to Korea Acute Myocardial Infarction Registry. Journal of Cardiology, 69 (1), 377-382. doi: http://doi.org/10.1016/j.jjcc.2016.09.002

12. Shiomi, H., Nakagawa, Y., Morimoto, T., Furukawa, Y., Nakano, A., Shirai, S. et. al. (2012). AS-015: Total Ischemic Time and Primary PCI: Optimal Time Period from Symptom-onset to Reperfusion Therapy. The American Journal of Cardiology, 109 (7), S7. doi: http://doi.org/10.1016/j.amjcard.2012.01.012

13. Ibanez, B., James, S., Agewall, S., Antunes, M. J., Bucciarelli-Ducci, C., Bueno, H. et. al. (2018). 2017 ESC Guidelines for the management of acute myocardial infarction in patients presenting with ST-segment elevation: The Task Force for the management of acute myocardial infarction in patients presenting with ST-segment elevation of the European So ciety of Cardiology (ESC). European Heart Journal, 39 (2), 119-177. doi: http://doi.org/10.1093/eurheartj/ehx393

14. Unifikovanyi klinichnyi protokol ekstrenoi, pervynnoi, vtorynnoi (spetsializovanoi) ta tretynnoi (vysokospetsializovanoi) medychnoi dopomohy ta medychnoi reabilitatsii «Hostryi koronarnyi syndrom z elevatsiieiu sehmenta ST» (2014). Nakaz MOZ Ukrainy No. 455. 02.07.2014. Kyiv: Derzhavnyi ekspertnyi tsentr, 7.

15. Venttsel, E. S., Ovcharov, L. A. (2000). Teoriya veroyatnostey i ee inzhenernye prilozheniya. Moscow: Vysshaya shkola, 480 .

16. Kolkutin, V. V., Pinchuk, P. V., Vasnetsova, O. A. (2005). About selection of experts for the analysis of competitiveness hardware-technical means for carrying out of judicial examinations. Problemy ekspertizy v meditsine, 5 (17), 5-8.

17. Novosad, V. P., Seliverstov, R. H., Artym, I. I. (2009). Kilkisni metody ekspertnoho otsiniuvannia. Kyiv: NADU, 36.

Received date 04.03.2021

Accepted date 15.04.2021

Published date 31.05.2021

Victor Zabashta, Director, Center of Emergency Care and Disaster Medicine, Nezalezhnosti ave., 13, Kharkiv, Ukraine, 61058

E-mail: healthco@med.edu.ua

Bogdan Fedak, Doctor of Medical Science, Professor, Department of Organization of Health Care, Public Administration, Kharkiv Medical Academy of Postgraduate Education, Amosova str., 58, Kharkiv, Ukraine, 61176 E-mail: fbs58@ukr.net 